\title{
Integrating Synchronous and Asynchronous Internet Distributed Education for Maximum Effectiveness
}

\author{
J. Mark Pullen \\ School of Information Technology and Engineering \\ George Mason University \\ Fairfax, Virginia 22030, USA \\ mpullen@gmu.edu \\ WWW: http://netlab.gmu.edu
}

\begin{abstract}
Distributed education delivered via the Internet is a growing practice, with most institutions offering at least course websites and many expanding to full course offerings and even degree offerings. There are two schools of thought with regard to delivery mode; the larger group has focused on asynchronous delivery, accessible at any time via webpages and interactive tutorials and quizzes, while a smaller group advocates synchronous delivery where students are online and interact during class time. This paper summarizes the advantages and disadvantages of the two delivery modes and describes our successful and growing experience of more than a decade using an open source synchronous delivery tool blended with a variety of asynchronous capabilities and classroom instruction. We conclude that a synergistic combination of the two modes with in-person instruction, designed to provide maximum flexibility to the student within the constraints of the subject, offers the best support for student learning.
\end{abstract}

\section{Introduction}

Distributed education via the Internet is a growing practice in many institutions today, making education more attainable by improving accessibility dramatically for students who experience barriers of schedule or distance to class attendance. Much of the education thus provided is based on asynchronous use of World Wide Web technology, where educational materials are accessed on the Internet via Web browser, primarily in text and graphic formats. This approach is simple to employ, although time consuming for the instructor, in that a collection of existing documents can form the core of an educational website. When the electronic documents are combined with an online learning management system supporting interactive tutorials, a qualitative improvement is achieved over the original form of 
asynchronous distance education, the paper-based correspondence course. Rapid growth in this area is described by Harris [1] and the Sloan Foundation [2].

While also using web sites and learning management systems heavily in our courses, the author and colleagues in the Volgenau School of Information Technology and Engineering at George Mason University (GMU) have pioneered use of synchronous Internet education as reported in [3] and [4]. For any given course session, roughly two-thirds of our students are in the classroom; the remainder are somewhere on the Internet, most often at home or office. We have described our teaching methodology, called simulteaching, and the supporting opensource technology, Network EducationWare, in [5] and [6]. Our approach extends a lecture or seminar over the Internet for improved accessibility by students. Goodwin and Bowman have shown that this approach results in the lowest costs for online teaching [7]. Moreover, we have found it results in the easiest transition from the traditional classroom, and is much more convenient to the student than distribution based on the other widely used synchronous approach, video teleconferencing.

Faculty involved in distributed education largely fall into two camps based on the dichotomy laid out above: synchronous or asynchronous. This paper addresses the fact that the two approaches are in fact highly complementary and should be used together in order to best support the learner. The paper begins with a description of our extensive work in synchronous Internet distributed education. This is followed by an assessment of the strengths and weaknesses of both synchronous and asynchronous modes, based on our practice in both areas. The assessment culminates in a discussion of the benefits of blending both approaches with traditional classroom instruction for maximum synergy.

\section{A Low-Cost Synchronous Delivery System and Its Capabilities}

While the author and colleagues have considerable experience in Web-based asynchronous distributed education, we are not unusual in that regard. Many educational programs provide such services, ranging in scope from simple course webpages to fully developed degree programs. Synchronous Internet delivery is less common and also offers great promise, as described by Wilson [8]. The perspective offered here for blending synchronous, asynchronous, and classroom delivery is based on our extensive experimentation using synchronous delivery, with which few educators have yet become familiar. Therefore this section begins with an overview of the supporting capability we have developed.

The author was privileged to conduct research using advanced networks for distributed military training in the early 1990s, at a time when the Internet was, in most cases, not capable of supporting synchronous interaction. These early experiments led to a conviction that nearly all the benefits of any social learning environment, from that of one-on-one-tutoring to that of large lecture halls, can be provided to remote participants using modern networking technology. As the capability of the Internet grew, this insight was applied to our courses at GMU. 
Beginning in 1995 we offered students the option of participating remotely in our classrooms. Initially we used Internet multicasting tools as described by Macedonia and Brutzman [9]. Pullen and Benson [4] reported on an early attempt at commercialization of technology to facilitate this type of interaction and the students' positive reaction to the ability to connect over dial-up modems to participate. More recently, our Network EducationWare (NEW) project has built on this paradigm to become a source of free software that facilitates expansion of this model by lowering the cost of support.

The NEW system software is represented in Figure 1 and summarized in this section. Executable and source code for all system components is openly available for academic purposes.

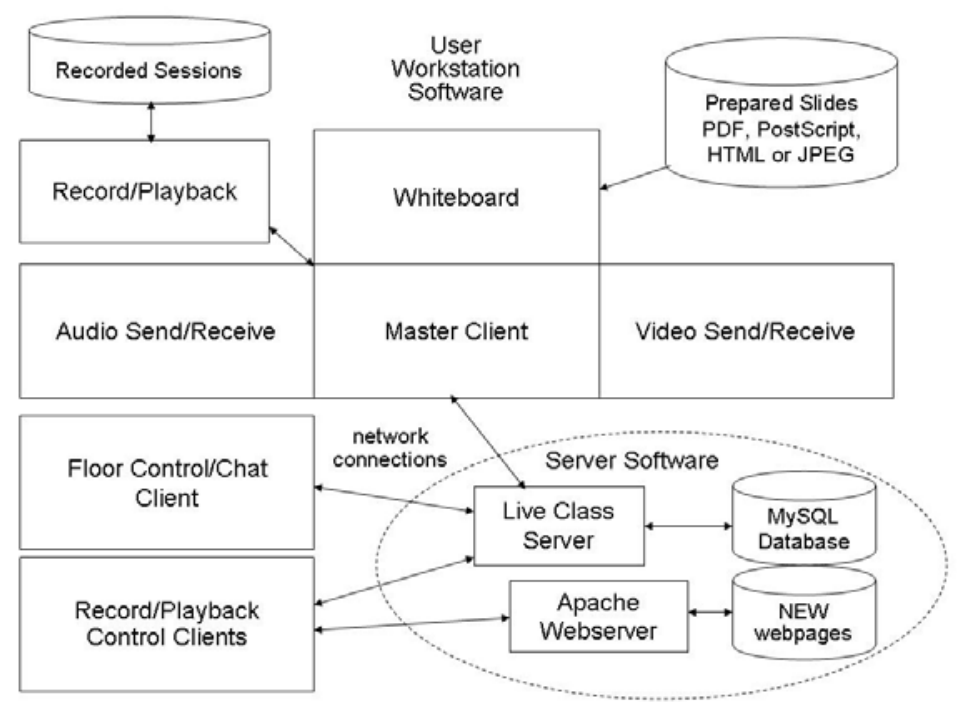

Fig. 1. NEW system software architecture

\subsection{Client Software}

The multimedia interface software in NEW derives from a variety of sources and runs on Windows and Linux-x86 platforms, with a Macintosh capability planned for late 2006.

We consider the Speak Freely Internet Audio software to be the most important component in the NEW system, both because it is essential to the students' learning experience and also because conveying voice with good quality over the Internet at low data rates presents a big challenge. SF is capable of passing good voice quality over the Internet, using a standard sound interface, and requiring only 20 kilobits per second of network capacity. We have added a graphic interface that provides all needed user functions in one easy-to-use package.

The Whiteboard provides the other key element for teaching online: graphics. It will display a precomposed graphic prepared in any of several open formats, 
including most importantly the Adobe Portable Document Format (PDF). The precomposed graphics can be annotated during class with lines, rectangles, ellipses, handwriting, and text in any color, a very useful feature for maintaining the attention of the visual learner. We prefer to use the whiteboard with a Tablet PC interface so that it becomes a surrogate chalkboard.

The optional Video tool is capable of multiple network formats, including standard H.323 conferencing. A typical delivery rate for NEW is two frames of 320 by 240 pixels per second, although rates up to 30 frames per second are possible. While we have found that, for teaching Information Technology, video provides a marginal benefit at relatively high cost, as reported in [10], we offer it as an option to students who have high quality Internet service.

The Master Client encapsulates data from the multicast applications into TCP tunnels to the Live Class server, prioritized according to the importance of each multimedia tool (audio first, whiteboard second, video last). The server can support a viable class connection over a $56 \mathrm{~kb} / \mathrm{s}$ modem, without video. The combination of clients and their network configuration established by the master client is controlled by a configuration file downloaded from the supporting webserver at the beginning of a NEW session. If software updates are indicated, the master client also downloads and installs them.

The NEW Floor Control shows the participants in the session, controls access to the virtual classroom "floor," provides for text questions to the instructor and text chat among the participants, and accepts URLs from the floor holder for browser launch on all participating client systems. It supports a "virtual hand raising" mode for lectures and an "anyone can have the floor" mode for seminars and meetings.

NEW Record and Playback Clients control their respective servers. They feature VCR-like button icons and an elapsed time readout. The playback control also is capable of jumping forward and backward to the next slide in the presentation. Recordings require about 5 Megabytes of disk per hour of class.

\subsection{Server Software}

The Live Class Server is the core of the NEW system. It implements group communication over the general Internet among a group of participating workstations by accepting a data stream combining transmissions from multicast conferencing tools on the floorholder's workstation and sending copies to all participants' workstations. It provides access control using either the system database or an external authentication service.

The Record and Playback servers are used to create and play streaming recordings that capture the information sent by NEW clients over the Internet from the instructor's workstation. Playback is accessed through the same software suite and also can be performed offline by downloading the recording files. Each segment of an online playback, starting with a new slide, can be Web-linked through a URL.

The NEW Course Management Webpages are the key to effective management of the mass of detail involved in supporting multiple courses. They provide teaching and learning functions, support and course management functions, authentication, and system administration functions. A single portal page provides access to all of 
these facilities, as described in [11]. Our webpages are hosted on an Apache Webserver that supports the PHP language needed for our webpages and a MySQL Database System supporting the standard Structured Query Language (SQL). Webbased support provides ubiquity and portability. It also makes possible data access over the network that we use to implement the chat room feature. The composite user interface for all tools is shown in Figure 2.

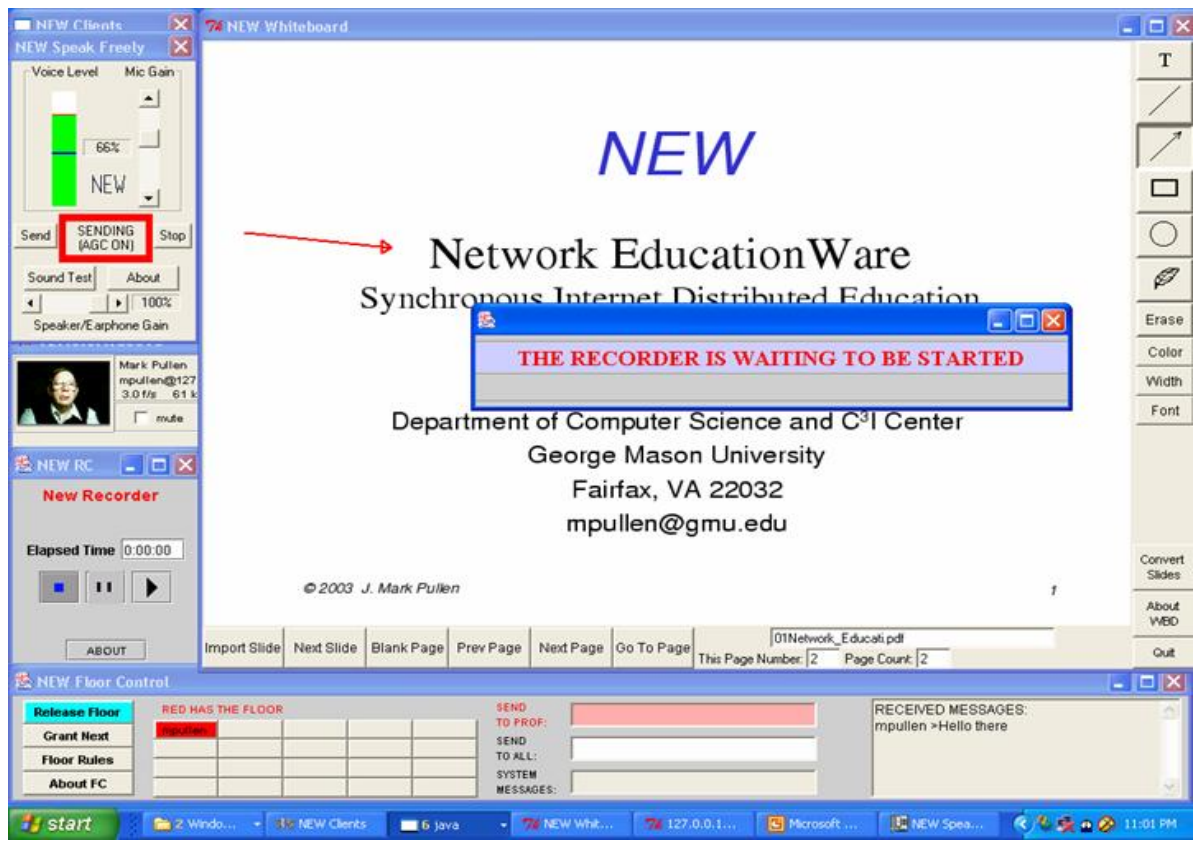

Fig. 2. NEW user interface for open-source synchronous teaching

\section{Characteristics of Synchronous Distributed Education}

The common style of classroom teaching in higher education today, while often criticized, actually represents a solution that has been optimized over time to offer the best mentoring to learners at a cost that is acceptable to the parties involved. The NEW system as described above is simply a virtual extension to that sort of classroom. As such, it inherits the advantages of a system that has been proved to work well over many years: An instructor, serving as interactive mentor and interpreter of course materials, can enable more effective use of students' time for learning and can do so cost-effectively by simultaneous presentation to a group. Specific characteristics of the synchronous mode are:

- Most information flow is from instructor to student, although students experience significant learning from each other and astute instructors often also learn from the students. Synchronous delivery supports instructor presentation extremely well. Quality of voice and graphic delivery is good to excellent. 
- A particularly important component of teaching is response to questions, because it clarifies points on which the students are confused and helps the instructor to refine the presentation. Synchronous delivery supports instructorstudent interaction well, by either voice communication or typed questions with voice response.

- However, unless every student has a microphone, in classroom and online, interaction among students is limited. We provide for typed questions during class and also support an always-available Web chat facility to help remedy this. Some faculty members hold office hours online in this way. Faculty and students also often supplement synchronous sessions with electronic mail (email).

- Faculty report that the time to prepare for class is increased very little when online students participate synchronously during scheduled classes. Most instructors today have developed graphic slides to support their presentations; these are readily converted to use with the shared whiteboard, and the instructor's pointer is replaced by whiteboard annotation tools.

- Simulteaching students online and the classroom allows economy of scale in that one presentation supports both, using a smaller classroom than the combined numbers would require. This does not mean that unlimited online students can be added, because faculty time for mentoring increases with the aggregate number. However, simulteaching not only reduces classroom costs but also allows low cost introduction of online teaching, despite limited initial online enrollment. Whereas it is generally agreed that asynchronous online education costs more than classroom education to provide, the low cost to introduce and savings in classroom facilities for simulteaching may actually reduce the cost to present a course.

- The author's experience in several courses has been that many students fail to reach timely completion when they do not have the motivation provided by regular course meetings. Regular synchronous meetings, whether online or in a classroom, result in fewer delayed student completions and dropouts.

- Recordings made synchronously support asynchronous delivery in quite a different way from websites. The recording captures much of the ambiance of the classroom that is promoted by instructor-student interaction. Moreover, in highly dynamic topics (including most Information Technology subjects), the presentation will include the latest developments which, of course, also are captured in the recording.

\section{Characteristics of Asynchronous Distributed Education}

The recordings described at the end of the previous section actually support the asynchronous mode, and are used as such in our program for both online and classroom students. Many of our students prize the flexibility to time-shift class sessions to suit their schedules, which adds a new dimension to accessibility of education. However, the benefit does not come without some costs. Benefits and drawbacks specific to the asynchronous mode are:

- Restating the point made above: the defining characteristic of the asynchronous mode, its freedom from schedule, can be a significant benefit, particularly to 
working students who must travel for their job. The Internet offers "any time, anywhere" access to learning resources.

- For students possessing the self-discipline to sustain self-study, no instructor or peers are necessary. These students are the natural customers for asynchronous distributed education. For them, pure "distance learning" is possible without the mentorship of an instructor.

- Peer interaction is difficult to impossible in the asynchronous environment. Even though an online chat room may be available for discussions, the fact that every student is likely to be studying a different topic greatly reduces the potential for discussion.

- Many students are not capable of totally independent study; they want and need access to human information resources. In the asynchronous mode, this is most often provided by email. The higher level of interaction available through an online chat room often proves useful, but with the schedule impact of "going synchronous” for an online session.

- Interactive tutorial materials available through a learning management system (LMS) such as WebCT, Blackboard, or the non-commercial Moodle system can be very effective resources for students. However, preparing such materials takes time and generating interactive materials can be extremely timeconsuming. Many faculty members, already carrying a full teaching load, have little interest in spending hundreds of hours to create interactive tutorials. As a result, institutions that want to offer the most effective asynchronous courses have found it necessary to offer release from teaching one or more courses for this purpose, entailing considerable expense.

- In a dynamic area such as Information Technology, once a course is posted online, it cannot be left without faculty attention. The subject area will continue to change so the course materials will need to be maintained.

\section{Asynchronous and Synchronous Modes Blended with Classroom Teaching}

At GMU we have steadily expanded the number of courses available synchronously; currently we offer twenty courses, taught by sixteen faculty members. Every faculty member approaches online delivery in an individual fashion. As the earliest of our faculty to begin teaching this way and the instructor for the largest number of different course offerings (six, to date), the author has had the opportunity to observe highly effective blended combinations. The following observations apply to the happy synergy of synchronous online, asynchronous online, and classroom education.

- The Web browser (e.g. Microsoft Internet Explorer; Mozilla Firefox) is far and away the most effective user interface for all Internet education. The ability of hypertext to link to the incredibly rich information resource of the World Wide Web also can be used to build links within an individual course or website. The browser can be used directly to offer text and graphics, or serve as a front end to other tools such as NEW or Adobe Acrobat.

- It is almost gratuitous to note that every course and every faculty member should have a webpage where course materials are posted and should interact 
with students at least daily by email. In addition, use of a chat room can provide for more interactive response, and is particularly valuable for students whose distance or schedule makes attending scheduled instructor office hours difficult or impossible.

- Given the large time commitment to develop them, it is not at all gratuitous but may be impractical to recommend development of interactive tutorials and homework/quizzes. These provide a highly effective means of review and assessment. We have created them for a few of our courses, and plan to link these directly to NEW recordings for immediate review where the student is having trouble in understanding. Another good practice, where suited to the subject, is an online project where the student creates something in a way that requires consideration of the principles taught in the course. In the Computer Science discipline, we are blessed with an abundance of such opportunities.

- As reported in [6], there has been no significant difference between the examination scores of classroom students and online students using NEW. We do not yet have a large enough sample to examine the degree to which availability of interactive tutorials and homework/quizzes has a direct impact on learning. However, we suspect such a study would yield similar results, because in our experience the primary impact of these mechanisms is to facilitate learning by increasing accessibility.

- Most students in our synchronous courses make an informed decision to trade the classroom experience for the considerable saving in commuting time that is enabled by Internet delivery. However, a small fraction of students report that they prefer online courses because they find the classroom environment distracting. We anticipate that this syndrome will be recognized by educational psychologists as online learning matures. Whatever the source, in our experience the online environment definitely benefits these students. Conversely, a larger fraction of students find the online environment impersonal and prefer classroom attendance, although many of these use the recordings for review. Thus, the best arrangement is to allow students maximum flexibility consistent with the course of study, so they may take advantage of the mode that best suits their needs.

- Simulteaching of classroom and synchronous online students is a highly effective approach with low costs and low barriers to adoption, using the arrangement shown in Figure 3. It is most effective when integrated with asynchronous supporting materials, but even if adopted without them it will result in increased accessibility to education over the Internet.

- When expanding a course to online delivery, consideration needs to be given to administrative support. Carswell has presented a compelling case to the effect that a true distance education program will allow students to do anything remotely [12]. However, we have found that a regional approach works well for us; our students come to campus for examinations unless special arrangements are made for proctoring. It would be possible, with some extra effort, to arrange for proctored online examinations via an LMS, conducted remotely at the testing centers offered by many community colleges today. In addition to testing, issues such as registration and textbook purchase must be considered. However, today the advent of Web-based administration and online bookstores removes most of the difficulty in these areas. 
- Above all else, any computer software the student is expected to use should be adaptable to as wide a range of platforms and operating systems as possible so it can be run on personal computers, taking the place of laboratory facilities.

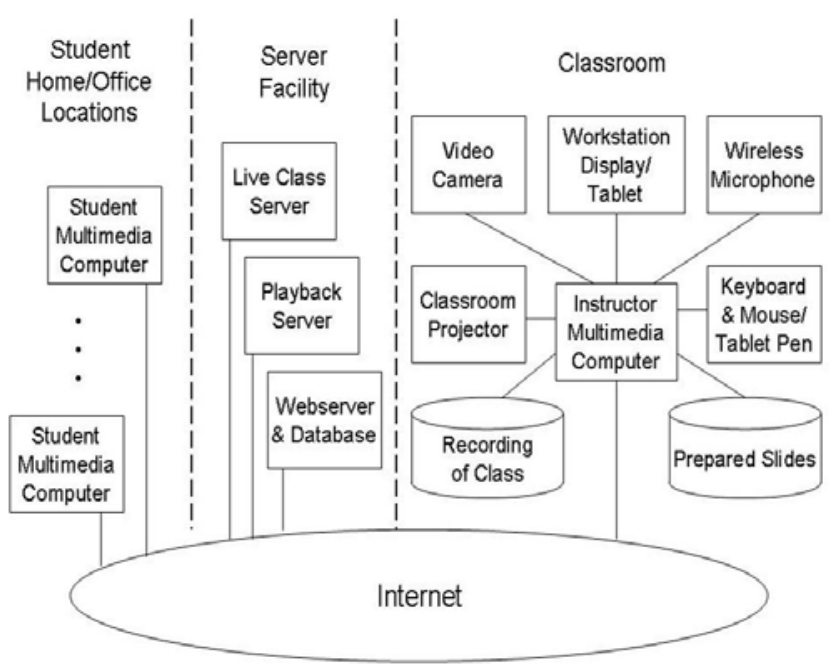

Fig. 3. Simulteaching combining classroom and the Internet

- The most important goal is accessibility for the student. Ideally each course that is suited to Internet delivery will be offered both in the classroom and synchronously online. It will include a variety of supporting asynchronous materials, with recordings of all class sessions readily available for online playback and download. Recordings will be integrated with tutorials and homework/quizzes in an LMS, and with supporting projects that can be accessed and submitted online. Any cost savings from facility reduction enabled by simulteaching ideally should be applied to support the effort required to integrate asynchronous materials. That process requires increased faculty time, but the payoff in support for the student is great.

\section{Conclusion}

Today's information technology offers support for teaching and learning that allows access to excellent education in many subjects from anywhere on the Internet. Synchronous Internet delivery offers improved accessibility to the student and is the simplest and least expensive to offer. Asynchronous Internet delivery provides high flexibility but its interactivity with the instructor and other students is poor and should be supplemented. The best way to employ these technologies is blending classroom instruction with synchronous online delivery by simulteaching, supporting the synchronous course with asynchronous Web-based resources, interactive tutorials, quizzes and homework, plus projects that can be completed or submitted online. Creating such a blended course with an effective set of 
asynchronous supporting materials that provides strong support and good flexibility for the student is challenging, but it is the best way to perform the academic mission given access to the Internet and its technologies.

\section{References}

1. D. Harris, Online Distance Education in the United States, in IEEE Communications 37(3), pp. 91-97 (1999)

2. Sloan Consortium, Sizing the Opportunity: The Quality and Extent of Online Education in the United States, 2002 and 2003, online at http://www.sloan-c.org/resources/overview.asp (2003)

3. J. Pullen, Synchronous Distance Education and the Internet, in Proceedings Internet Society Annual Conference, Geneva, Switzerland, published online at http://www.isoc.org/inet98/ proceedings/4b/4b_1.htm (1998)

4. J. Pullen and M. Benson, ClassWise: Synchronous Internet Desktop Education, November 1999 special multimedia CDROM issue of IEEE Transactions on Education 42(4); printed abstract 370 (1999)

5. J. Pullen and P. McAndrews, Low-Cost Internet Synchronous Distance Education Using Open-Source Software, Proceedings of the ASEE Annual Conference 2004, Salt Lake City, UT, (June 2004)

6. C. Snow, J. Pullen and P. McAndrews, An Open-Source Web-Based System for Synchronous Distance Education, in IEEE Transactions on Education 48(4), 705-712 (2005)

7. C. Goodwin, and M. Bowman, Is the bottom line of online out of line? Calculating the total cost of online courses in Technology Curricula, in Proceedings ASEE Annual Conference 2004, Salt Lake City, UT, (June 2004)

8. J. Wilson, After the Fall: Lessons of an Indulgent Era, plenary presentation, Distance Education 2003, University of Wisconsin, unpublished, available online at http://www.jackmwilson.com/ArticlesTalks/eLearning-Wisconsin2003.ppt (2003)

9. M. Macedonia and D. Brutzman , Mbone Provides Audio and Video Across the Internet, in IEEE Computer 27(4), pp. 30-36 (April 1994)

10. J. Pullen, Applicability Of Internet Video In Distance Education For Engineering, in Proceedings IEEE Frontiers in Education 2001, Reno, NV, T2F-14-T2F-19, online at http://fie.engrng.pitt.edu/ fie2001/papers/1242.pdf (October 2001)

11. J. Pullen and P. McAndrews, A Web Portal For Open-Source Synchronous Distance Education, IASTED Journal on Advanced Technology for Learning 2(1) (January 2005)

12. L. Carswell, The "Virtual University": Toward an Internet Paradigm? in ACM SIGCSE Bulletin 30(3), pp. 46-50 (September 1998) 